\title{
On the Coverage Process of a Moving Target in a Dynamic Nonstationary Sensor Field
}

\author{
Pallavi Manohar and D. Manjunath \\ Department of Electrical Engg., IIT Bombay \\ Mumbai INDIA \\ email: pallavim, dmanjudee.iitb.ac.in
}

\begin{abstract}
We analyze the statistical properties of the $k$ coverage of a point-target moving in a straight line in a dynamic, nonstationary sensor field. The availability of each node is modeled by an independent, $\{0,1\}$-valued continuous time Markov chain. Sensor locations form a nonhomogeneous spatial Poisson process. The sensing areas of the sensors are circles of i.i.d. radii. We first describe the induced nonstationary MarkovBoolean model and obtain $k$-coverage of the target at an arbitrary time instant. We then obtain $k$-coverage statistics for the time interval $[0, T]$. A pointwise stationary approximation that yields a limit theorem is also discussed. Numerical results illustrate the analysis.
\end{abstract}

\section{INTRODUCTION}

Coverage is a canonical problem in the design and analysis of wireless sensor networks. If the sensor locations and their sensing areas are known, a deterministic coverage analysis typically based on computational geometry techniques can be performed. If the sensor locations and the sensing areas are known only via their statistical properties, e.g., distributions, a stochastic analysis based on techniques from stochastic geometry is performed. In this paper, our interest is in the latter. Most stochastic coverage analysis e.g.,[1], [2], [3], is based on the techniques developed in [4], a classic introduction to the theory of coverage processes.

Let $P=\left\{\zeta_{1}, \zeta_{2}, \ldots\right\}$ be a countable collection of points in $d$-dimensional Euclidean space and $\left\{C_{1}, C_{2}, \ldots\right\}$ a countable collection of nonempty $d$-dimensional sets. Then, $\mathcal{C}=\left\{\zeta_{i}+\right.$ $\left.C_{i}: i=1,2, \ldots\right\}$ is a coverage process [4]. If $P$ is a stationary Poisson point process and the $C_{i}$ 's are i.i.d. random sets, independent also of $P$, then $\mathcal{C}$ is called a Boolean model.

It is now a standard practice in coverage analysis of sensor networks to model the sensor field as a Boolean model: the sensor locations form a Poisson point process and the sensing areas are the random sets. Our first point of departure from the standard assumptions is that we allow $P$ to be a nonstationary Poisson process. Of course, we can also allow the $C_{i}$ to depend on $\zeta_{i}$. This leads us to define the following three types of nonstationary Boolean models. If $P$ is a nonstationary Poisson point process, and the $C_{i}$ are i.i.d. then $\mathcal{C}$ will be called a typeA nonstationary or nonhomogeneous Boolean model. If $P$ is a stationary Poisson point process and the $C_{i}$ are independent but not identically distributed (the distribution depending on the location of $\zeta_{i}$ ), then $\mathcal{C}$ will be called a type-B nonstationary

-Pallavi Manohar is supported by a Philips Research Fellowship.
Boolean model. The type-AB nonstationary Boolean model is now obvious: $P$ is a nonstationary Poisson point process and the $C_{i}$ are not identically distributed. In this paper we will be concerned with type-A and type-AB nonstationary Boolean models.

Our second point of departure from the usual analyses is that we consider a dynamic coverage process. At any time, a sensor may or may not be available for coverage. This can be due to many reasons.

1) A sensor can alternate between active and sleep (a low power operational state) to conserve energy. A sleep scheduling algorithm determines the transitions between the two states. The schedule could be based on relative power levels at the sensor nodes or to provide a specified coverage characteristic.

2) A sensor might be outside the communication range of its neighbours due to channel fading.

3) In a mobile sensor network, the sensor might move to a different location.

A $\{0,1\}$-valued continuous time stationary Markov chain is used to model the availability of each sensor $-\mathrm{a}$ ' 1 ' indicating that it is available and a ' 0 ' indicating that it is not available. The availability of a sensor is independent of the availability of the other sensors. Thus, our interest is in the coverage process $\mathcal{C}_{t}:=\left\{Y_{i}^{t}\left(\zeta_{i}+C_{i}\right): i=1,2, \ldots\right\}$ where, $\left\{Y_{i}^{t}\right\}_{t \in \Re}$, $i=1,2, \ldots$, are independent, $\{0,1\}$-valued, continuous time stationary Markov chains. The process $\mathcal{C}_{t}$ will be called the Markov-Boolean model of Type-A nonstationarity. In [7], a similar model has been used for a homogeneous sensor network with uncoordinated power saving mechanisms and is called the blinking Poisson Boolean model. It is shown that any message generated by a sensor will reach the sink node in time proportional to the distance between the sensor and the sink, using dynamic percolation theory.

In this paper, we will analyse the coverage process of a point target moving on a straight line path in a two-dimensional sensor field modeled by $\mathcal{C}_{t}$ defined above. Most target tracking applications require that $k$ or more sensors 'cover' a target to estimate its position with $k \geq 3$ being common. In this paper we will be analysing the $k$-coverage-coverage by at least $k$-sensors-of the moving target. Analysis of the coverage of one-dimensional paths in two-dimensional fields has been considered in [5], [6]. In [5], $P$ is assumed to be a homogeneous Poisson process and the $C_{i}$ are i.i.d. In [6], the 


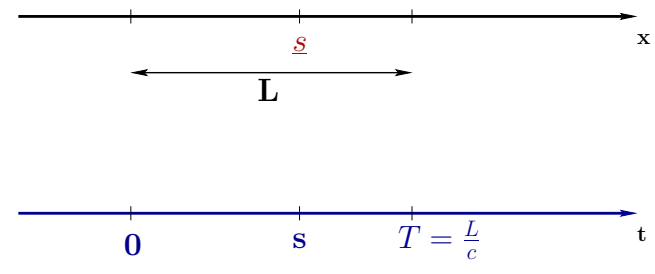

Fig. 1. Time and space axes for the target moving on the $x$-axis with velocity $c$ during the time interval $[0, T]$

sensors are assumed to be available continuously while $\mathcal{C}$ is a type-A nonstationary Boolean model.

The rest of the paper is organized as follows. In Section II, the coverage process of a target moving with constant velocity is described and the probability of $k$-coverage of the target at any time instant is obtained. Then we study $k$-coverage of the target in the time interval $[0, T]$. The main results are developed in Section III. We obtain the expectation and variance of the duration for which the target is not $k$-covered in the interval $[0, T]$. Next, we provide a simple approximation to obtain the fraction of time for which the target is not $k$-covered during $[0, T]$. We then obtain bounds on the $k$-coverage of the target over the entire time interval. Numerical illustration of the key results are provided for the 'Laplacian' deployment. We conclude the paper with a discussion in Section IV.

\section{PRELIMINARIES}

Consider a target moving on a straight line in $\Re^{2}$ with a constant velocity of $c$ units per second. Without loss of generality, we will assume that the target is moving on the $x$-axis. Let $\underline{s}:=c \times s$ be the position of the target on the $x$-axis at time $s$. Thus, the coverage of the target at time $s$ is essentially the coverage of the target at the location $\underline{s}$ on the $x$-axis by the active sensors at time $s$ (Fig. 1).

Let us now consider the state of a sensor. Let $\mu_{0}$ be the transition rate from the 0 -state (sleep state) to the 1-state (active state) and $\mu_{1}$ the transition rate from 1-state to the 0 -state. The stationary probability of the sensor being in state $j, p_{j}$, is given by $p_{j}=\frac{\mu_{1-j}}{\mu_{1}+\mu_{0}}, j \in\{0,1\}$. Define the timedependent transition probability between the states as follows: $p_{t}(j, k):=\operatorname{Pr}\left(Y^{s+t}=k \mid Y^{s}=j\right)$ for $j, k \in\{0,1\}$. It can be shown that

$$
p_{t}(j, j)=\left(1-p_{j}\right) e^{-\eta t}+p_{j}
$$

where $\eta=\mu_{0}+\mu_{1}$ and $p_{t}(j, k)=1-p_{t}(j, j)$ for $j, k \in\{0,1\}$ and $j \neq k$.

Let us now consider the coverage process on the $x$-axis by a two-dimensional, type-A nonhomogeneous Boolean model. Here we assume that all the sensors are active. Let $\lambda(x, y)$ be the two-dimensional Poisson intensity function for the sensor locations. Sensor $i$ is assumed to cover a circle of random radius $R_{i}, R_{i}$ are i.i.d. with the distribution having support in $[0,1]$. It can be shown that the coverage of the $x$-axis by the two-dimensional field can be analyzed by considering an equivalent one-dimensional type- $\mathrm{AB}$ nonhomogeneous Boolean model [6]. This model is parametrized by $\bar{\lambda}(x)$, the intensity function of the one-dimensional nonhomogeneous
Poisson point process and $\bar{R}_{x}$, the location-dependent random coverage length. The coverage by a point at $x_{1}$ on the $x$-axis will be the segment $\left(x_{1}-\bar{R}_{x_{1}}, x_{1}+\bar{R}_{x_{1}}\right)$ of the $x$-axis. Let $f_{\bar{R}_{x}}(\bar{r})$ denote the probability density function of $\bar{R}_{x}$. The following is shown in [6].

$$
\begin{aligned}
\bar{\lambda}(x) & =\int_{0}^{1} \int_{0}^{r}(\lambda(x, y)+\lambda(x,-y)) d y f_{R}(r) d r \\
f_{\bar{R}_{x}}(\bar{r}) & =\left\{\begin{array}{c}
\frac{\bar{r}}{a(x) \beta(x)} \int_{\bar{r}}^{1} \frac{f_{R}(r)}{\sqrt{r^{2}-\bar{r}^{2}}}\left(\lambda\left(x, \sqrt{r^{2}-\bar{r}^{2}}\right)+\right. \\
\left.\lambda\left(x,-\sqrt{r^{2}-\bar{r}^{2}}\right)\right) d r \text { for } 0 \leq \bar{r} \leq 1 \\
0
\end{array}\right.
\end{aligned}
$$

Here

$a(x):=\int_{-1}^{1} \lambda(x, y) d y$ and

$\beta(x):=\operatorname{Pr}\left(C_{i}\right.$ of sensor with abscissa $x$ intersects $x$-axis $)$. If $\lambda(x, y)$ can be written as $\lambda_{1}(x) \lambda_{2}(y)$, then $\bar{R}_{x}$ is independent of $x$ and $\left\{\bar{R}_{i}\right\}$ are i.i.d., otherwise they are independent but not identically distributed.

Our interest is in the $k$-coverage of the moving point target by the dynamic coverage process on the $x$-axis described above with the sensor nodes switching their states. Define $\psi_{k}(s)$ as follows

$$
\psi_{k}(s)= \begin{cases}1 & \text { if target is not } k \text {-covered at time } s, \\ 0 & \text { otherwise },\end{cases}
$$

i.e., $\psi_{k}(s)$ is the indicator that the point $\underline{s}$ on the $x$-axis is not $k$-covered by the active sensors at time $s$. Clearly, the number of sensors that cover a point $\underline{s}$ on the $x$-axis is Poisson distributed and its mean $m(\underline{s})$, is given by

$$
m(\underline{s})=\int_{\underline{s}-1}^{\underline{s}+1} \bar{\lambda}(u) p_{1} \gamma(\underline{s}, u) d u .
$$

Here $\gamma(\underline{s}, u)$ is the probability that point $\underline{s}$ is covered by a sensor at location $u$, i.e., $\gamma(\underline{s}, u)=\operatorname{Pr}\left(\bar{R}_{u} \geq|\underline{s}-u|\right)$. Hence,

$$
\operatorname{Pr}\left(\psi_{k}(s)=1\right)=\sum_{j=0}^{k-1} \frac{m(\underline{s})^{j} e^{-m(\underline{s})}}{j !},
$$

The coverage of the target at time $s$ depends only on the stationary probability $p_{1}$ and not on the time dependent transition probabilities.

Let $V_{T, k}$ be the random variable denoting the duration for which the target is not $k$-covered in the time interval $[0, T]$, i.e.,

$$
V_{T, k}:=\int_{0}^{T} \psi_{k}(s) d s .
$$

In the next section we obtain the moments of $V_{T, k}$.

\section{III. $k$-COVERAGE OF THE TARGET IN TIME INTERVAL $[0, T]$}

Expectation and variance of $V_{T, k}, \mathrm{E}\left(V_{T, k}\right)$ and $\operatorname{VAR}\left(V_{T, k}\right)$, respectively, are obtained as below. $\mathrm{E}\left(V_{T, k}\right)$ is straightforward and is given by

$$
\mathrm{E}\left(V_{T, k}\right)=\int_{0}^{T}\left(\sum_{j=0}^{k-1} \frac{m(\underline{s})^{j} e^{-m(\underline{s})}}{j !}\right) d s .
$$




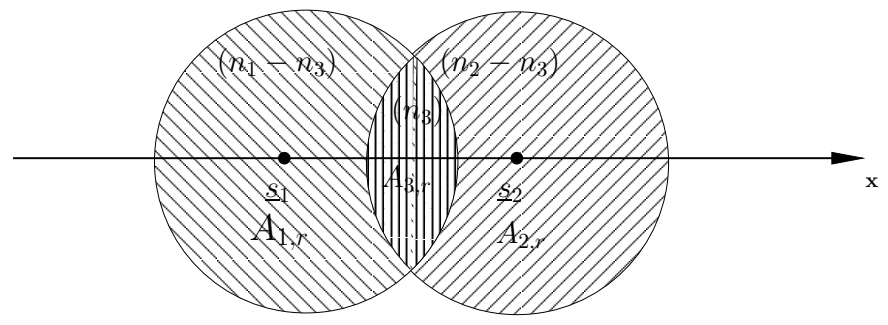

Fig. 2. Coverage of points $\underline{s}_{1}$ and $\underline{s}_{2}$ by sensors of coverage radius $r$. Sensors with radius $r$ in $A_{1, r}$ cover only $\underline{s}_{1}$, those in $A_{2, r}$ cover only $\underline{s}_{2}$ and those in $A_{3, r}$ cover both $\underline{s}_{1}$ and $\underline{s}_{2}$. There are $\left(n_{1}-n_{3}\right),\left(n_{2}-n_{3}\right)$ and $n_{3}$ sensors with sensing radius $r$ in the regions $A_{1, r}, A_{2, r}$ and $A_{3, r}$ respectively.

It can be shown that $\operatorname{VAR}\left(V_{T, k}\right)$ satisfies the relation

$$
\operatorname{VAR}\left(V_{T, k}\right)=\int_{0}^{T} \int_{0}^{T} \operatorname{CoV}\left(\psi_{k}\left(\underline{s}_{1}\right) \psi_{k}\left(\underline{s}_{2}\right)\right) d s_{1} d s_{2}
$$

Since $\psi_{k}(\cdot)$ is an indicator variable, we can write

$$
\begin{gathered}
\operatorname{COV}\left(\psi_{k}\left(\underline{s}_{1}\right) \psi_{k}\left(\underline{s}_{2}\right)\right)=\operatorname{Pr}\left(\underline{s}_{1} \text { and } \underline{s}_{2} \text { not } k \text {-covered }\right) \\
-\operatorname{Pr}\left(\underline{s}_{1} \text { not } k \text {-covered }\right) \operatorname{Pr}\left(\underline{s}_{2} \text { not } k \text {-covered }\right) .
\end{gathered}
$$

To help us evaluate (8) we use the following informal argument that can be made rigorous. Consider the sensors with sensing radius $r$. Of these sensors, let $E_{a, r}, E_{b, r}$, and $E_{c, r}$, respectively be the set of sensors that cover only $\underline{s}_{1}$, only $\underline{s}_{2}$ and both. The size of these sets is independent because for a fixed $r$, these sensors come from nonoverlapping regions of $\Re^{2}$. Fig. 2 illustrates this. $E_{a, r}$ is the set of sensors with sensing radius $r$ and located in the region $A_{1, r}$ in Fig. 2. Let $E_{a}:=\cup_{0 \leq r \leq 1} E_{a, r}$. Clearly, $\left|E_{a}\right|$ is Poisson with expectation $\int_{0}^{1} \int_{A_{1, r}} \lambda(u, v) d u d v d r$. Similarly, $\left|E_{b}\right|$ and $\left|E_{c}\right|$ are also Poisson. Further, $E_{a, r_{1}}, E_{b, r_{2}}$, and $E_{c, r_{3}}$ are also independent for any $r_{1}, r_{2}$, and $r_{3}$. Thus $E_{a}, E_{b}$, and $E_{c}$ are also independent.

The second term in (8) is determined from (4). To evaluate the first term we make use of the above argument as follows.

- Consider the segment of the $x$-axis $\left[\underline{s}_{1}, \underline{s}_{2}\right]$ and the set of sensors that cover both $\underline{s}_{1}$ and $\underline{s}_{2}$, or equivalently, the sensors that cover the moving target at time instants $s_{1}$ and $s_{2}$. Let $\alpha\left(\underline{s}_{1}, \underline{s}_{2}\right)$ be the mean number of such sensors. Defining $\hat{\gamma}\left(\underline{s}_{1}, \underline{s}_{2}, u\right)$ to be the probability that the moving target is covered by a sensor at $u$ at both the points $\underline{s}_{1}$ and $\underline{s}_{2}$, we obtain

$$
\begin{gathered}
\hat{\gamma}\left(\underline{s}_{1}, \underline{s}_{2}, u\right):=\operatorname{Pr}\left(\bar{R}_{u} \geq \max \left(\left|\underline{s}_{2}-u\right|,\left|\underline{s}_{1}-u\right|\right)\right) \\
\alpha\left(\underline{s}_{1}, \underline{s}_{2}\right)=\int_{\underline{s}_{2}-1}^{\underline{s}_{1}+1}\left(\bar{\lambda}(u) p_{1} p_{s_{2}-s_{1}}(1,1)\right. \\
\left.\hat{\gamma}\left(\underline{s}_{1}, \underline{s}_{2}, u\right)\right) d u
\end{gathered}
$$

- Let $\tilde{m}\left(\underline{s}_{1}, \underline{s}_{2}\right)$ be the mean number of sensors covering the target at point $\underline{s}_{1}$ but not at $\underline{s}_{2}$. Defining $\tilde{\gamma}\left(\underline{s}_{1}, \underline{s}_{2}, u\right)$ to be the probability that a sensor at $u$ covers the target at point $\underline{s}_{1}$ and not at $\underline{s}_{2}$ we get

$$
\begin{aligned}
& \tilde{\gamma}\left(\underline{s}_{1}, \underline{s}_{2}, u\right):=\operatorname{Pr}\left(\left|\underline{s}_{1}-u\right| \leq \bar{R}_{u}<\left|\underline{s}_{2}-u\right|\right), \\
& \tilde{m}\left(\underline{s}_{1}, \underline{s}_{2}\right)=\int_{\underline{s}_{1}-1}^{\underline{s}_{1}+1}\left(\bar{\lambda}(u) p_{1} \tilde{\gamma}\left(\underline{s}_{1}, \underline{s}_{2}, u\right)\right) d u+ \\
& \int_{\underline{s}_{2}-1}^{\underline{s}_{1}+1}\left(\bar{\lambda}(u) p_{1} p_{s_{2}-s_{1}}(1,0) \hat{\gamma}\left(\underline{s}_{1}, \underline{s}_{2}, u\right)\right) d u(10)
\end{aligned}
$$

- Similarly, let $\tilde{m}\left(\underline{s}_{2}, \underline{s}_{1}\right)$ be the mean number of sensors covering the target at point $\underline{s}_{2}$ but not at $\underline{s}_{1}$ and $\tilde{\gamma}\left(\underline{s}_{2}, \underline{s}_{1}, u\right)$ be the probability that a sensor at $u$ covers the target at point $\underline{s}_{2}$ and not at $\underline{s}_{1}$. We thus get

$$
\begin{aligned}
\tilde{\gamma}\left(\underline{s}_{2}, \underline{s}_{1}, u\right) & :=\operatorname{Pr}\left(\left|\underline{s}_{2}-u\right| \leq \bar{R}_{u}<\left|\underline{s}_{1}-u\right|\right), \\
\tilde{m}\left(\underline{s}_{2}, \underline{s}_{1}\right) & =\int_{\underline{s}_{2}-1}^{\underline{s}_{2}+1}\left(\bar{\lambda}(u) p_{1} \tilde{\gamma}\left(\underline{s}_{2}, \underline{s}_{1}, u\right)\right) d u+ \\
\int_{\underline{s}_{2}-1}^{\underline{s}_{1}+1} & \left(\bar{\lambda}(u) p_{0} p_{s_{2}-s_{1}}(0,1) \hat{\gamma}\left(\underline{s}_{1}, \underline{s}_{2}, u\right)\right) d u .(11)
\end{aligned}
$$

We can now obtain the probability that $\underline{s}_{1}$ and $\underline{s}_{2}$ are not $k$-covered as follows.

$$
\begin{gathered}
\operatorname{Pr}\left(\underline{s}_{1} \text { and } \underline{s}_{2} \text { not } k \text {-covered }\right)=\sum_{n_{1}, n_{2}=0}^{k-1} \sum_{n_{3}=0}^{\min \left(n_{1}, n_{2}\right)} \\
\left.\left(n_{2}-n_{3}\right) \text { cover } \underline{s}_{2}, \text { not } \underline{s}_{1} ; n_{3} \text { cover both } \underline{s}_{1} \text { and } \underline{s}_{2}\right) \\
=\sum_{n_{1}, n_{2}=0}^{k-1} \sum_{n_{3}=0}^{\min \left(n_{1}, n_{2}\right)} \\
\left(\frac{e^{-\tilde{m}\left(\underline{s}_{1}, \underline{s}_{2}\right)}\left(\tilde{m}\left(\underline{s}_{1}, \underline{s}_{2}\right)\right)^{\left(n_{1}-n_{3}\right)}}{\left(n_{1}-n_{3}\right) !}\right) \times \\
\\
\left(\frac{e^{-\tilde{m}\left(\underline{s}_{2}, \underline{s}_{1}\right)}\left(\tilde{m}\left(\underline{s}_{2}, \underline{s}_{1}\right)\right)^{\left(n_{2}-n_{3}\right)}}{\left(n_{2}-n_{3}\right) !}\right) \times \\
\\
\left(\frac{e^{-\alpha\left(\underline{s}_{1}, \underline{s}_{2}\right)}\left(\alpha\left(\underline{s}_{1}, \underline{s}_{2}\right)\right)^{\left(n_{3}\right)}}{\left(n_{3}\right) !}\right) .
\end{gathered}
$$

The last step follows as the three component events in the RHS of the first equality above are independent.

The variance of $V_{T, k}$ can be obtained from (4), (7), (8) and (12). Note that the effect of transitions of $\left\{Y^{t}\right\}$ is captured in the variance of $V_{T, k}$ through $\alpha\left(\underline{s}_{1}, \underline{s}_{2}\right), \tilde{m}\left(\underline{s}_{1}, \underline{s}_{2}\right)$, and $\tilde{m}\left(\underline{s}_{2}, \underline{s}_{1}\right)$.

We now provide a numerical illustration of the above results. Let $\lambda(x, y)=\kappa e^{-\theta(|x|+|y|)}$ be the intensity function of the nonhomogeneous Poisson process with $\kappa>0$ and $\theta>0$ where $\theta$ is the decay parameter. We refer to this as the 'Laplacian' deployment because of its similarity with the Laplacian distribution. We will also assume that $C_{i}$ are disks of unit radius. From (1) and (2) we obtain,

$$
\begin{aligned}
\bar{\lambda}(x) & =\frac{2 \kappa\left(1-e^{-\theta}\right)}{\theta} e^{-\theta|x|}, \\
f_{\bar{R}}(\bar{r}) & =\frac{\bar{r} e^{-\theta\left(\sqrt{1-\bar{r}^{2}}\right)}}{\frac{\left(1-e^{-\theta}\right)}{\theta} \sqrt{1-\bar{r}^{2}}} .
\end{aligned}
$$

The expressions for $m(x)$ and the first order statistics $\operatorname{Pr}\left(\psi_{k}(s)\right)$ (recall that $\psi_{k}(s)$ is an indicator variable) and 


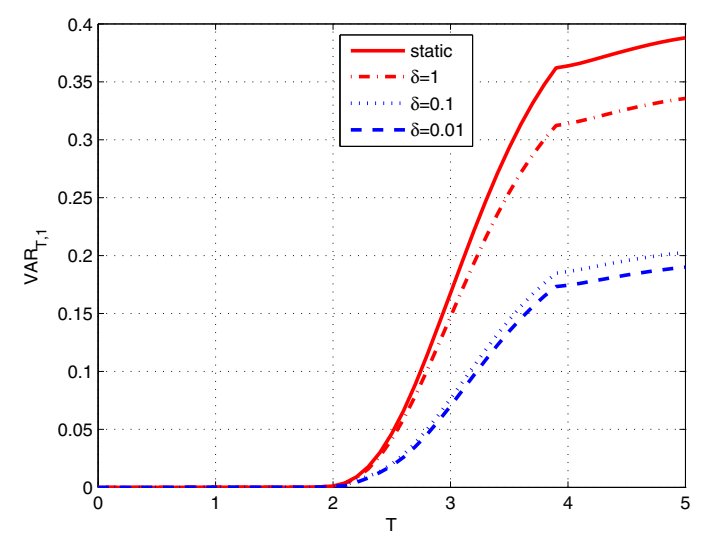

Fig. 3. Variance of $V_{T, 1}$ (for the target moving on the $x$-axis with unit velocity i.e., $c=1$ ) is plotted as a function of $T$ for different $\delta$ with $\theta=$ $2, \kappa=100, \mu_{1}=4, \mu_{0}=6$. Here only transition rates of the Markov chain are scaled by $\frac{1}{\delta}$.

$\mathrm{E}\left(V_{T, k}\right)$ for the 'Laplacian' deployment can be easily obtained as these are the same as that for the static network obtained in [6] but with the Poisson density thinned by $p_{1}$, i.e., with intensity $\left(p_{1} \lambda(x)\right)$.

The variance of $V_{T, k}$ depends on the transition rates of the Markov chain determining the availability of a sensor. To study the effect of transition rates on the variance of $V_{T, k}$, we scale the transition rates of the Markov chain by $1 / \delta$. The stationary probability of the Markov chain is independent of the scaling, i.e., $p_{i}(\delta)=p_{i} \forall \delta$ and the scaled time-dependent transition probabilities will be $p_{s}^{\delta}(j, j)=\left(1-p_{j}\right) e^{-(\eta / \delta) s}+p_{j}$. The variance is plotted as a function of $T$ for different $\delta$ in Figs. 3 (for $k=1$ ) and 4 (for $k=2$ ) where $\mu_{1}=4, \mu_{0}=6, \theta=2$ and $\kappa=100$ so that the expected number of sensors to be deployed is kept constant. Recall that $\eta=\mu_{1}+\mu_{0}$ which is equal to 10 and is also scaled by the factor $\frac{1}{\delta}$.

For $\delta=\infty$, the sensors are either in active or sleep state and do not have state transitions. This corresponds to the static sensor network with Poisson density $p_{1} \lambda(x)$. Notice that this has the highest variance for $V_{T, k}$. As $\delta \rightarrow 0$, the variance decreases monotonically and has a nonzero limit. The limit can be obtained with $\alpha\left(\underline{s}_{1}, \underline{s}_{2}\right), \tilde{m}\left(\underline{s}_{1}, \underline{s}_{2}\right)$, and $\tilde{m}\left(\underline{s}_{2}, \underline{s}_{1}\right)$ given by

$$
\begin{aligned}
\alpha\left(\underline{s}_{1}, \underline{s}_{2}\right)= & \int_{\underline{s}_{2}-1}^{\underline{s}_{1}+1}\left(\bar{\lambda}(u) p_{1}^{2} \hat{\gamma}\left(\underline{s}_{1}, \underline{s}_{2}, u\right)\right) d u \\
\tilde{m}\left(\underline{s}_{1}, \underline{s}_{2}\right)= & \int_{\underline{s}_{1}-1}^{\underline{s}_{1}+1}\left(\bar{\lambda}(u) p_{1} \tilde{\gamma}\left(\underline{s}_{1}, \underline{s}_{2}, u\right)\right) d u+ \\
& \int_{\underline{s}_{2}-1}^{\underline{s}_{1}+1}\left(\bar{\lambda}(u) p_{1} p_{0} \hat{\gamma}\left(\underline{s}_{1}, \underline{s}_{2}, u\right)\right) d u \\
\tilde{m}\left(\underline{s}_{2}, \underline{s}_{1}\right)= & \int_{\underline{s}_{2}-1}^{\underline{s}_{2}+1}\left(\bar{\lambda}(u) p_{1} \tilde{\gamma}\left(\underline{s}_{2}, \underline{s}_{1}, u\right)\right) d u+ \\
& \int_{\underline{s}_{2}-1}^{\underline{s}_{1}+1}\left(\bar{\lambda}(u) p_{1} p_{0} \hat{\gamma}\left(\underline{s}_{1}, \underline{s}_{2}, u\right)\right) d u
\end{aligned}
$$

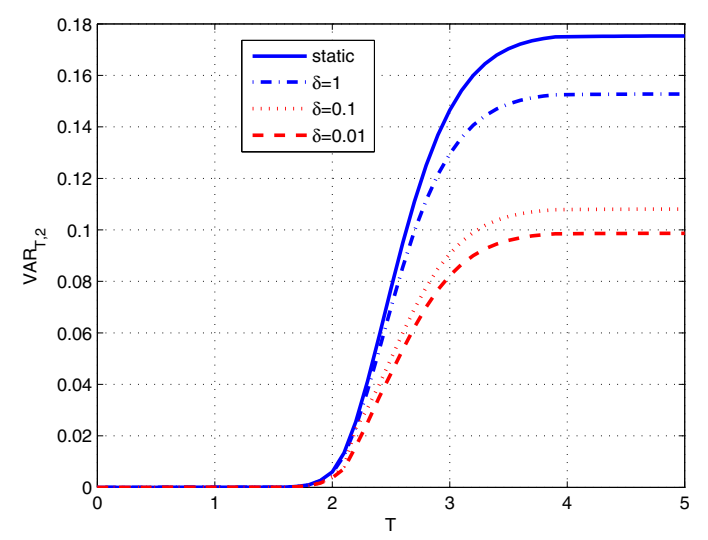

Fig. 4. Variance of $V_{T, 2}$ (for the target moving on the $x$-axis with unit velocity i.e., $c=1$ ) is plotted as a function $T$ for different $\delta$ with $\theta=2, \kappa=$ $100, \mu_{1}=4, \mu_{0}=6$. Here only transition rates of the Markov chain are scaled by $\frac{1}{\delta}$.

\section{A. An Approximation and a Scaling Law for the $k$-coverage}

We have obtained exact expressions for the first and second moments of $V_{T, k}$. However, the expressions are messy and do not have closed forms. We seek a 'pointwise stationary approximation' (PSA) [9] for $\phi_{k}(T):=V_{T, k} / T$. PSA has been used in the analysis of queueing systems with nonstationary arrival and service processes. Let $\chi\left(s_{1}, s_{2}\right)$ be a time average coverage statistic of interest in the interval $\left(s_{1}, s_{2}\right), s_{1}<s_{2}$, in the nonstationary Markov-Boolean model. The PSA of $\chi\left(s_{1}, s_{2}\right)$, denoted by $\chi^{(P S A)}\left(s_{1}, s_{2}\right)$ is defined as

$$
\chi^{(P S A)}\left(s_{1}, s_{2}\right):=\frac{1}{s_{2}-s_{1}} \int_{s_{1}}^{s_{2}} \tilde{\chi}(s) d s
$$

where $\tilde{\chi}(s)$ is the stationary statistic $\chi$ when the point process is a stationary process with the intensity, $\bar{\lambda}(\underline{s})$ and the coverage radii, $\bar{R}(\underline{s})$. This is an attractive approximation because, typically $\tilde{\chi}(s)$ has a closed form and the above can be easily evaluated. For example, for $\left(\phi_{k}(T)\right.$ the PSA can be written as

$$
\phi_{k}(T) \approx \phi_{k}(T)^{(P S A)}=\frac{1}{T} \int_{0}^{T} \tilde{\phi}_{k}(d s),
$$

where $\tilde{\phi}_{k}(d s)$ is the fraction of time for which the target is not being covered in a stationary Markov-Boolean model with Poisson process $\bar{\lambda}(\underline{s})$ and coverage radius distribution as that of $\bar{R}(\underline{s})$.

In [10] it has been shown that the PSA is asymptotically exact for the one-dimensional nonhomogeneous Boolean model of type-AB. This provides us with a weak law of large numbers for the fraction of the segment on the $x$-axis that is $k$-covered. We now derive a similar scaling law for the onedimensional Markov-nonhomogeneous Boolean model (type$\mathrm{AB}$ ) that shows that the PSA for the fraction of time the target is not $k$-covered is also asymptotically exact.

Let $\mathcal{C}_{t}(\delta)$ be the process $\mathcal{C}_{t}$ with Poisson intensity $(\bar{\lambda}(\underline{s}) / \delta)$, the random coverage radius $\delta \bar{R}(\underline{s})$ and the transition rates of the Markov chain $\mu_{i} / \delta$.

Theorem 1: For the scaled nonhomogeneous one dimensional Markov-Boolean process, $\mathcal{C}_{t}(\delta)$, the fraction of time in 
the time interval $[0, T]$, for which the target is not $k$-covered, denoted by $\phi_{k}^{\delta}(T)$, converges to the PSA in probability, i.e., as $\delta \rightarrow 0$,

$$
\begin{gathered}
\phi_{k}^{\delta}(T) \stackrel{\mathrm{p}}{\longrightarrow} \sum_{l=0}^{k-1} \frac{1}{T} \int_{0}^{T}\left(\frac{\left(\bar{\lambda}(\underline{s}) p_{1} \mathrm{E}(\bar{R}(\underline{s}))\right)^{l}}{l !}\right. \\
e^{\left.-\bar{\lambda}(\underline{s}) p_{1} \mathrm{E}(\bar{R}(\underline{s}))\right) d s}
\end{gathered}
$$

Proof: The arguments are similar to that for the nonhomogeneous Boolean model derived in [10] and we only provide a sketch of the proof.

It is clear that $p_{1}$ is invariant to the scaling of $\mu_{0}$ and $\mu_{1}$. From (3), the expected number of sensors sensing the target at time $s$ (at location $\underline{s}$ ), depends only on $p_{1}$ and not on the time-dependent transition probabilities; hence it is invariant to the scaling of transition rates.

Consider the coverage of target during the interval $[s-\epsilon, s+$ $\epsilon]$. Let $\chi_{k}^{\delta}(s, \epsilon)$ be the fraction of time in $[s-\epsilon, s+\epsilon]$, for which the target is not $k$-covered. Let $\bar{\lambda}_{\text {sup }}(s, \epsilon)$ be the supremum of $\bar{\lambda}(x), x \in[\underline{s}-\epsilon, \underline{s}+\epsilon]$ and $\bar{R}_{\text {sup }}(s, \epsilon)$ a 'stochastic supremum' of $\bar{R}(x), x \in[\underline{s}-\epsilon, \underline{s}+\epsilon]$. (A stochastic supremum of a set of random variables is a random variable that dominates all the elements of the set.) Let $\chi_{k \text {,sup }}(s, \epsilon)$, denotes fraction of time for which the target is not $k$-covered in a stationary MarkovBoolean model with Poisson intensity $\bar{\lambda}_{\text {sup }}(s)$ and coverage radius $\bar{R}_{\text {sup }}(s)$. Let $\chi_{k \text {,sup }}^{\delta}(s, \epsilon)$, be the corresponding statistic in the scaled process $\mathcal{C}_{t}(\delta)$. It can be shown that

$$
\begin{aligned}
\lim _{\delta \rightarrow 0} \chi_{k, \text { sup }}^{\delta}(s, \epsilon)= & \sum_{l=0}^{k-1}\left(e^{-\bar{\lambda}_{\text {sup }}(s, \epsilon) p_{1} \mathrm{E}\left(\bar{R}_{\text {sup }}(s, \epsilon)\right)} \times\right. \\
& \left.\frac{\left(\bar{\lambda}_{\text {sup }}(s, \epsilon) p_{1} \mathrm{E}\left(\bar{R}_{\text {sup }}(s, \epsilon)\right)\right)^{l}}{l !}\right) .
\end{aligned}
$$

We can similarly define $\chi_{k \text {,inf }}(s, \epsilon)$, and $\chi_{k, \text { inf }}^{\delta}(s, \epsilon)$. We have defined $\chi_{k \text {,sup }}(s, \epsilon)$, and $\chi_{k \text {,inf }}(s, \epsilon)$, so that they form stochastic upper and lower bounds on $\chi_{k}^{\delta}(s, \epsilon)$.

When $\epsilon \rightarrow 0, \delta \rightarrow 0$ such that $\frac{\epsilon}{\delta} \rightarrow \infty$ we can show that

$$
\chi_{k}^{\delta}(s, \epsilon) \stackrel{\mathrm{d}}{\longrightarrow} \sum_{l=0}^{k-1} \frac{\left(\bar{\lambda}(\underline{s}) p_{1} \mathrm{E}(\bar{R}(\underline{s}))\right)^{l} e^{-\bar{\lambda}(\underline{s}) p_{1} \mathrm{E}(\bar{R}(\underline{s}))}}{l !}
$$

From the definition of $\chi_{k}^{\delta}(s, \epsilon)$ and since $\chi_{k}^{\delta}(s, \epsilon) \leq 1$, by using dominated convergence theorem we can write,

$$
\lim _{\delta \rightarrow 0} \phi_{k}^{\delta}(T)=\frac{1}{T} \int_{0}^{T} \lim _{\substack{\delta \rightarrow 0 \\ \epsilon \rightarrow 0}} \chi_{k}^{\delta}(s, \epsilon) d s .
$$

From (15), (16) we get

$$
\begin{gathered}
\lim _{\delta \rightarrow 0} \phi_{k}^{\delta}(T)=\sum_{l=0}^{k-1} \frac{1}{T} \int_{0}^{T}\left(\frac{\left(\bar{\lambda}(\underline{s}) p_{1} \mathrm{E}(\bar{R}(\underline{s}))\right)^{l}}{l !} \times\right. \\
e^{\left.-\bar{\lambda}(\underline{s}) p_{1} \mathrm{E}(\bar{R}(\underline{s}))\right) d s,}
\end{gathered}
$$

in distribution. As convergence in distribution to a constant implies convergence in probability the theorem follows.

Let us now discuss what happens to the process $\mathcal{C}_{t}(\delta)$ as $\delta$ goes to zero.

1) When $\delta$ is large, the state transitions of the sensors have no effect on the coverage process. This is because

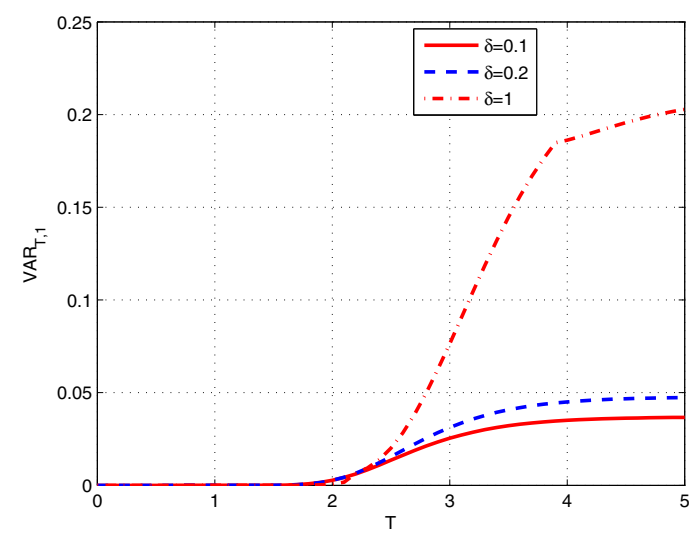

Fig. 5. Variance of $V_{T, 1}$ for the target moving on the $x$-axis with unit velocity i.e., $c=1$ is plotted as a function $T$ for different values of $\delta$ with $\theta=2, \kappa=100, \mu_{1}=4, \mu_{0}=6$. This plot is for the scaled process $\mathcal{C}_{t}(\delta)$ i.e. Poisson intensity, random coverage radius, and transition rates of the Markov chain are scaled.

the scaled transition rates $\frac{\mu_{1}}{\delta}$ and $\frac{\mu_{0}}{\delta}$ are small. The sensors in the area $A_{3,1}$ do not change state as the target moves from $\underline{s}_{1}$ to $\underline{s}_{2}$. This is similar to the case of large $\delta$ studied earlier in the section. Thus $\alpha\left(\underline{s}_{1}, \underline{s}_{2}\right), \tilde{m}\left(\underline{s}_{1}, \underline{s}_{2}\right), \tilde{m}\left(\underline{s}_{2}, \underline{s}_{1}\right)$, and hence the variance depend only on $p_{1}$.

$$
\begin{aligned}
\alpha\left(\underline{s}_{1}, \underline{s}_{2}\right) & =\int_{\underline{s}_{2}-1}^{\underline{s}_{1}+1}\left(\bar{\lambda}(u) p_{1} \hat{\gamma}\left(\underline{s}_{1}, \underline{s}_{2}, u\right)\right) d u, \\
\tilde{m}\left(\underline{s}_{1}, \underline{s}_{2}\right) & =\int_{\underline{s}_{1}-1}^{\underline{s}_{1}+1}\left(\bar{\lambda}(u) p_{1} \tilde{\gamma}\left(\underline{s}_{1}, \underline{s}_{2}, u\right)\right) d u, \\
\tilde{m}\left(\underline{s}_{2}, \underline{s}_{1}\right) & =\int_{\underline{s}_{2}-1}^{\underline{s}_{2}+1}\left(\bar{\lambda}(u) p_{1} \tilde{\gamma}\left(\underline{s}_{2}, \underline{s}_{1}, u\right)\right) d u(18)
\end{aligned}
$$

2) As $\delta$ becomes small only the stationary probability of a sensor being active matters. There is a further thinning of the Poisson density by $p_{1}$. This corresponds to the nonzero limit for the variance which can be obtained using (13).

3) For is $0<\delta<\infty$, the stationary as well as the timedependent transition probabilities affect the variance and $\alpha\left(\underline{s}_{1}, \underline{s}_{2}\right), \tilde{m}\left(\underline{s}_{1}, \underline{s}_{2}\right), \tilde{m}\left(\underline{s}_{2}, \underline{s}_{1}\right)$ are given by (9), (10), and (11) respectively.

We numerically study the variance for different $\delta$. It is observed that the variance of $V_{T, k}$ decreases as $\delta$ is decreased. Decreasing $\delta$ effectively increases $\eta$ (the sum of the transition rates) and hence the number of transitions per unit time. As $\delta$ is decreased, the behaviour of the variance for the scaled process $\mathcal{C}_{t}(\delta)$ is similar to the case when only the transition rates are scaled. However, the value of variance is significantly lower when the transition rates, the Poisson density and coverage radii are all scaled together, see Fig. 5 where all the three parameters are scaled and Fig. 3 where only transition rates are scaled. 


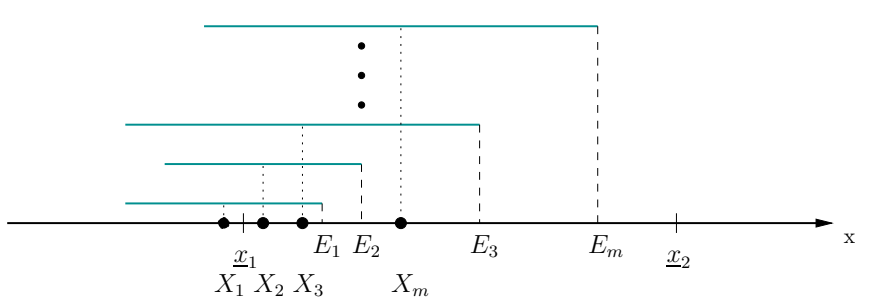

Fig. 6. The dots correspond to the Poisson points (active sensors) covering $\underline{x}_{1}$ denoted as $\left\{X_{i}\right\}$. The line segments show the extent of the coverage of the $x$-axis by the $\left\{X_{i}\right\}$, i.e., they mark the intervals $\left(X_{i}-\bar{R}_{X_{i}}, X_{i}+\bar{R}_{X_{i}}\right)$. The ordered sequence of endpoints, $E_{1}, \ldots, E_{m}$ for the point $\underline{x}_{1}$ is also marked.

\section{B. Complete $k$-coverage of the target in time interval $\left[x_{1}, x_{2}\right]$}

We now study the probability that the target is $k$-covered over the entire time interval $\left[x_{1}, x_{2}\right]$ denoted by $T$, i.e., $\operatorname{Pr}\left(V_{T, k}=0\right)$. This is the probability of complete $k$-coverage of the segment $\left[\underline{x}_{1}, \underline{x}_{2}\right]$ on the $x$-axis traced by the target during the time interval $\left[x_{1}, x_{2}\right]$ as seen from Fig. 1. We obtain upper and lower bounds on the complete $k$-coverage of the target in time interval $\left[x_{1}, x_{2}\right]$.

An upper bound on $V_{T, k}$ can be obtained from the expectation and the variance of $V_{T, k}$ from (6) and (7) respectively and is given by,

$$
\operatorname{Pr}\left(V_{T, k}=0\right) \leq \frac{\operatorname{VAR}\left(V_{T, k}\right)}{\operatorname{VAR}\left(V_{T, k}\right)+\left(\mathrm{E}\left(V_{T, k}\right)\right)^{2}}
$$

We now obtain lower bounds using a method analogous to that in [11]. We first make following definitions.

- Let the point $\underline{s}$ on the $x$-axis be covered by $m$ active sensors. Consider the segments of the $x$-axis traced by the target that are covered by these active sensors and label the endpoints of these segments as $E_{i}, i=1, \ldots, m$ such that $E_{1} \leq E_{2} \leq \ldots \leq E_{m}$. See Fig. 6 for an illustration. Define $\omega_{k}(\underline{s})$ as follows.

$$
\omega_{k}(\underline{s})= \begin{cases}E_{m-k+1} & \text { if } \psi_{k}(s)=0 \text { i.e., } m \geq k, \\ \underline{s} & \text { if } \psi_{k}(s)=1 \text { i.e., } m<k .\end{cases}
$$

- Consider the sequence $Z_{k}^{(n)}\left(\underline{x}_{1}\right)$, parametrized by $\underline{x}_{1}$ and indexed by $n$, defined as follows.

$$
\begin{aligned}
Z_{k}^{(1)}\left(\underline{x}_{1}\right) & :=\omega_{k}\left(\underline{x}_{1}\right) \\
Z_{k}^{(n)}\left(\underline{x}_{1}\right) & :=\omega_{k}\left(Z_{k}^{(n-1)}\left(\underline{x}_{1}\right)\right), \quad n \geq 2 \\
Z_{k}^{(\infty)}\left(\underline{x}_{1}\right) & :=\sup _{n \geq 1} Z_{k}^{(n)}\left(\underline{x}_{1}\right)
\end{aligned}
$$

Observe that $Z_{k}^{(\infty)}\left(\underline{x}_{1}\right)$ corresponds to the point at which the $k$-covered segment starting at $\underline{x}_{1}$ ends. (If $\underline{x}_{1}$ is not $k$ covered then, from the definition, the segment 'ends' at $\underline{x}_{1}$.) The distribution of $Z_{k}^{(\infty)}\left(\underline{x}_{1}\right)$ determines the probability of complete $k$-coverage of the segment $\left[\underline{x}_{1}, \underline{x}_{2}\right]$, i.e.,

$$
\operatorname{Pr}\left(V_{T, k}=0\right)=\operatorname{Pr}\left(Z_{k}^{(\infty)}\left(\underline{x}_{1}\right)>\underline{x}_{2}\right)
$$

The distribution of $Z_{k}^{(1)}\left(\underline{x}_{1}\right)$ i.e., of $\omega_{k}\left(\underline{x}_{1}\right)$, can be obtained from the probability that there are less than $k$ active sensors each of which covers the target over the entire segment $\left[\underline{x}_{1}, \underline{x}_{2}\right]$. Let $\beta\left(\underline{s}_{1}, \underline{s}_{2}\right)$ be the mean number of active sensors each of which covers the entire segment $\left[\underline{s}_{1}, \underline{s}_{2}\right]$ and is given by

$$
\begin{aligned}
\hat{\gamma}\left(\underline{s}_{1}, \underline{s}_{2}, u\right):=\operatorname{Pr}\left(\bar{R}_{u} \geq \max \left(\left|\underline{s}_{2}-u\right|,\left|\underline{s}_{1}-u\right|\right)\right), \\
\beta\left(\underline{s}_{1}, \underline{s}_{2}\right)=\int_{\underline{s}_{2}-1}^{\underline{s}_{1}+1} \bar{\lambda}(u) p_{1}\left(1-\int_{0}^{s_{2}-s_{1}} \mu_{1} e^{-\mu_{1} t} d t\right) \\
\times \hat{\gamma}\left(\underline{s}_{1}, \underline{s}_{2}, u\right) d u .
\end{aligned}
$$

The tail distribution of $\omega_{k}\left(\underline{x}_{1}\right)$, obtained using $\beta\left(\underline{x}_{1}, \underline{x}_{2}\right)$, is a lower bound as we consider only the sensors covering the segment $\left[\underline{x}_{1}, \underline{x}_{2}\right]$ which are active over the entire time interval $\left[x_{1}, x_{2}\right]$.

$\operatorname{Pr}\left(\omega_{k}\left(\underline{x}_{1}\right)>\underline{x}_{2}\right) \geq 1-\left(\sum_{j=0}^{k-1} e^{-\beta\left(\underline{x}_{1}, \underline{x}_{2}\right)} \frac{\beta\left(\underline{x}_{1}, \underline{x}_{2}\right)^{j}}{j !}\right)$

Along the lines of the analysis in [12], we obtain the following lower bound for $\operatorname{Pr}\left(V_{T, k}=0\right)$,

$$
\begin{aligned}
\operatorname{Pr}\left(V_{T, k}=0\right) & =\operatorname{Pr}\left(Z_{k}^{(\infty)}\left(\underline{x}_{1}\right)>\underline{x}_{2}\right) \\
& \geq \operatorname{Pr}\left(Z_{k}^{(n)}\left(\underline{x}_{1}\right)>\underline{x}_{2}\right) \quad \forall n \geq 1 .
\end{aligned}
$$

Tighter lower bounds can be obtained by using larger values of $n$. In [11], the sequence $Z_{k}^{(n)}\left(\underline{x}_{1}\right)$ is shown to have Markov property for the static nonhomogeneous Boolean model with $k=1$. For the Markov nonhomogeneous Boolean model with, $k \geq 1$, we can see that the sequence does not have the Markov property. Thus for Markov nonstationary Boolean model $\operatorname{Pr}\left(Z_{k}^{(n)}\left(\underline{x}_{1}\right)>\underline{x}_{2}\right)$ is difficult to obtain for general $n$ and the approach in [11] cannot be used. However, we can obtain the distribution of $Z_{k}^{(2)}\left(\underline{x}_{1}\right)$ as follows [12].

$$
\begin{array}{r}
\operatorname{Pr}\left(Z_{k}^{(2)}\left(\underline{x}_{1}\right)>\underline{x}_{2}\right)=\operatorname{Pr}\left(Z_{k}^{(1)}\left(\underline{x}_{1}\right)>\underline{x}_{2}\right)+ \\
\int_{\underline{x}_{1}}^{\underline{x}_{2}}\left(\operatorname{Pr}\left(Z_{k}^{(2)}\left(\underline{x}_{1}\right)>\underline{x}_{2} \mid Z_{k}^{(1)}\left(\underline{x}_{1}\right)=z_{1}\right) \times\right. \\
\left.\operatorname{Pr}\left(Z_{k}^{(1)}\left(\underline{x}_{1}\right) \in d z_{1}\right)\right) .
\end{array}
$$

We can obtain only a lower bound on $\operatorname{Pr}\left(Z_{k}^{(2)}\left(\underline{x}_{1}\right)>\underline{x}_{2}\right)$ using (20). Thus the lower bounds obtained for the Markov nonhomogeneous Boolean model are loose compared to that for the static model [12].

We numerically study the upper and lower bounds for the 'Laplacian' deployment. Fig. 7 shows upper and the lower bounds obtained with $n=1$ and $n=2$ from transition rates of $\mu_{1}=4, \mu_{0}=6$. Observe that the lower bound with $n=2$ is loose. This bound can be improved by decreasing the state transition rates. For example with $\mu_{1}=0.04, \mu_{0}=0.06$ the lower bound is tighter as shown in Fig. 8. This is because the RHS of (20) approaches the tail distribution on LHS as $\mu_{1} \rightarrow 0$. The lower bound with $n=2$ is quite tight for the static $\left(\mu_{1}=0\right)$ nonhomogeneous Boolean model [12]. 


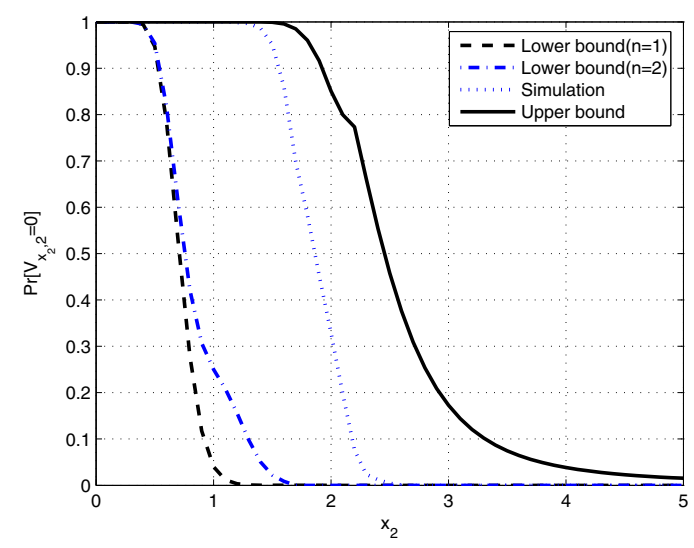

Fig. 7. Bounds on the probability of complete 2-coverage of the target in the time interval $\left[0, x_{2}\right]$ is plotted as a function $x_{2}$ with $\theta=2, \kappa=100, \mu_{1}=$ $4, \mu_{0}=6, c=1$.

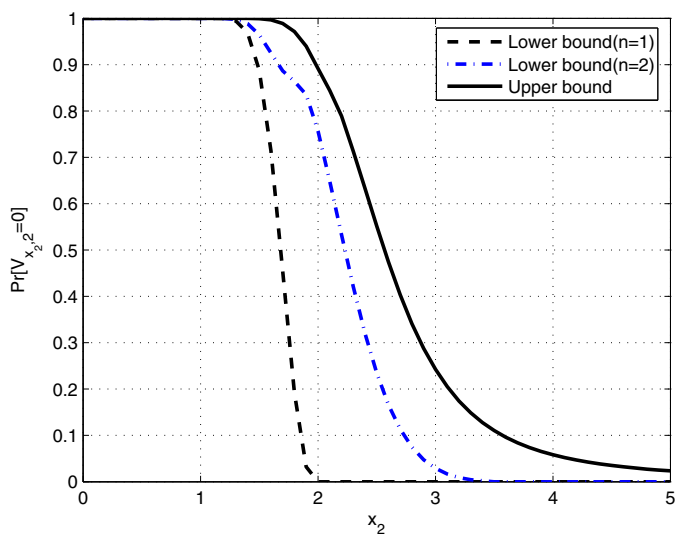

Fig. 8. Bounds on the probability of complete 2-coverage of the target in the time interval $\left[0, x_{2}\right]$ is plotted as a function $x_{2}$ with $\theta=2, \kappa=100, \mu_{1}=$ $0.04, \mu_{0}=0.06, c=1$.

\section{Discussion}

We have presented the analysis of $k$-coverage of the target, moving in a straight line with a constant velocity, by a Markovnonhomogeneous Boolean (type-A) sensor field. Two reasons motivate this analysis. First, non homogeneity can be a natural consequence of the deployment process. Second, like in the 'Laplacian' density example, the expected number of sensors can be made finite. Thus, this analysis can be a proxy to analyse the coverage when a finite number of nodes are deployed.

We have obtained the probability of $k$-coverage of the target at a time instant $s$, the expected duration of for which the target is not $k$-covered over $[0, T]$ and its variance. Since these expressions are rather messy and do not have closed form, we also consider the simple pointwise stationary approximation. A scaling law shows that the PSA for the Markovnonhomogeneous Boolean model is asymptotically correct. The effect of scaling of the time-dependent transition rates on the variance is studied as the effect of such scaling is captured only in the variance. We have also obtained bounds on the $k$ - coverage of the target over the entire time interval $[0, T]$.

We have used a simple two state Markov chain to model temporal behaviour of sensors. This can be extended to a three state Markov chain with an absorbing state called failure corresponding to the failure of a sensor node. Every sensor node will eventually die because of complete loss of battery power. This feature is captured by the model with the absorbing state. Thus the coverage analyses using this model should essentially cover the network evolution over the entire time horizon. We need to solve the absorbing Markov chain to obtain transition probabilities which is quite involved.

The state transition rates $\mu_{1}, \mu_{0}$ are dictated by the sleep scheduling algorithm implemented in the sensor network. The rates essentially determine the temporal behavior of the target coverage. Thus it is interesting to determine the transition rates for the desired expected coverage time of the target i.e., to provide a sleep scheduling algorithm for the random sensor deployment under the Markov-nonhomogeneous Boolean model. One of the methods to obtain the transition rates is estimation of the rates using observations of the induced coverage on a straight line.

\section{ACKNOWLEDGEMENT}

We would like to thank the referees for their insightful comments, especially for (10) and (11). This research was carried out in Bharati Centre for Communication Research.

\section{REFERENCES}

[1] S. Kumar, T. H. Lai, and J. Balogh, "On $k$-coverage in a mostly sleeping sensor network," in Proceedings of ACM MobiCom, September/October 2004.

[2] B. Liu and D. Towsley, "A study on the coverage of large-scale sensor networks," in Proceedings of 1st IEEE International Conference on Mobile Ad-hoc and Sensor Systems, 2004.

[3] P. J. Wan and C. W. Yi, "Coverage by randomly deployed wireless sensor networks," IEEE/ACM Transactions on Networking, vol. 14, pp. 2658-2669, 2006

[4] P. Hall, Intoduction to the Theory of Coverage Process. John Wiley and Sons, 1988.

[5] S. K. Iyer, D. Manjunath, and D. Yogeshwaran, "Limit laws for $k$ coverage of paths by a Markov-Boolean model," Submitted, 2007.

[6] P. Manohar, S. S. Ram, and D. Manjunath, "On the path coverage by a non homogeneous sensor field," in Proceedings of IEEE Globecom, 2006.

[7] O. Dousse, P. Mannersalo, and P. Thiran, "Latency of wireless sensor networks with uncoordinated power saving mechanisms," in Proceedings of the 5th ACM MobiHoc, 2004, pp. 109-120.

[8] S. M. Ross, Stochastic Processes, 2nd ed. John Wiley and Sons, 1996.

[9] L. Green and P. Kolesar, "The pointwise stationary approximation for queues with nonstationary arrivals," Management Science, vol. 37, pp. 84-97, 1991.

[10] S. S. Ram, D. Manjunath, and V. B. Borkar, "A note on busy period statistics for $\mathrm{M}_{t} / \mathrm{G}_{t} / \infty$ queues," Submitted, 2006

[11] W. Stadje, "Coverage problems for random intervals," SIAM Journal on Applied Mathematics, vol. 49, no. 5, pp. 1538-1551, October 1989.

[12] P. Manohar, S. S. Ram, and D. Manjunath, "Path coverage by a sensor field : The non homogeneous case," Submitted, 2007. 